\title{
Características físico-químicas e químicas e estudo preliminar de estabilidade de tinturas preparadas com espécies de arnica Lychnophora em comparação com Arnica montana
}

\author{
Renata L. Maciel ${ }^{1}$, Lígia M. Moreira-Campos ${ }^{2}$, Breno C. Silva $^{2}$, Maria G. Lins Brandão ${ }^{*}$
}

${ }^{1}$ Laboratório de Farmacognosia, Departamento de Produtos Farmacêuticos, Faculdade de Farmácia, Universidade

Federal de Minas Gerais, Av. Antônio Carlos, 6627, 31270-901, Belo Horizonte, MG, Brasil

${ }^{2}$ Laboratório de Controle de Qualidade de Produtos Farmacêuticos e Cosméticos, Departamento de Produtos

Farmacêuticos, Faculdade de Farmácia, Universidade Federal de Minas Gerais, Av. Antônio Carlos, 6627,

31270-901, Belo Horizonte, MG, Brasil

\begin{abstract}
RESUMO: Arnica é o nome originalmente atribuído à espécie Arnica montana L.(Asteraceae), planta de origem Européia e utilizada em várias partes do mundo. A medicina tradicional brasileira atribui o nome de arnica também a outras espécies da família Asteraceae, especialmente algumas do gênero Lychnophora. No presente estudo foram determinadas as características físico-químicas (organoléptica, teor alcoólico, densidade, $\mathrm{pH}$, porcentagem de resíduo seco) e químicas (CCD e CLAE) de tinturas preparadas com as arnicas L. pinaster e L. rupestris, em comparação com a $A$. montana. As amostras foram então submetidas a estudo de estabilidade, a partir da sua permanência em estufa climatizada por seis meses e em prateleira por 10 meses. Os resultados demonstraram alguma semelhança entre as tinturas de espécies de Lychnophora e a A. montana, especialmente entre seus perfis em CCD. Todas as tinturas sofreram alterações após o período de permanência na estufa, sendo o mais significante a redução no valor dos teores de resíduos secos, indicando degradação das substâncias e perda por volatilização. O conjunto das análises permitiu distinguir as tinturas de cada uma das espécies e confirmou a necessidade de determinar prazos de validade para as mesmas, devido à sua a instabilidade ao longo do tempo.
\end{abstract}

Unitermos: Lychnophora, Arnica montana, arnica, tintura, qualidade, estabilidade.

\begin{abstract}
Physico-chemical and chemical characteristics and stability study of tincture from the arnicas Lychnophora and Arnica montana". Arnica is the name attributed to Arnica montana L. (Asteraceae), an european species used in several parts of the world. The Brazilian traditional medicine also attributes the name of arnica to other species of the Asteraceae, especially some Lychnophora. In the present study physical-chemical characteristics (organoleptic, alcoholic graduation, density, $\mathrm{pH}$, percentage of dry residue) and chemical (TLC and HPLC profiles) of tinctures prepared with $L$. rupestris, L. pinaster and A. montana has been determined. The samples had been then submitted the thermal stability testing. The results demonstrated some similarity between tinctures of the species of Lychnophora and A. montana, especially by their profiles in CCD. All tinctures altered after six months by thermal stability testing, being the most significant the reduction of percentage of dry residues, indicating degradation and loss of substances. The results allowed to distinguish tincture from the different species of arnica and confirmed their instability on the time.
\end{abstract}

Keywords: Lychnophora, Arnica montana, arnica, tincture, quality, stability.

\section{INTRODUÇÃO}

Arnica montana L. (Asteraceae) é uma espécie nativa da Europa extensivamente utilizada na medicina tradicional daquele continente no tratamento de contusões, inflamações, dores musculares e reumáticas (Blumenthal, 1998). A droga vegetal, constituída pelos capítulos florais secos, encontra-se inscrita em Farmacopéias de vários países, inclusive nas três primeiras edições da Farmacopéia
Brasileira (Farmacopéia, 1926; 1959; 1977). Estudos em animais confirmaram a atividade antiinflamatória de extratos da planta, sendo esta atribuída à presença de lactonas sesquiterpênicas do tipo helenalina (Hall et al., 1979; Lyss et al., 1997). Essa atividade está relacionada à inibição da ativação do fator de transcripção NF kappa $\beta$ (Rungeler et al., 1999; Klass et al., 2002). As lactonas sesquiterpênicas desencadeiam também ação citotóxica 
contra hepatócitos humanos (Woerdenbag et al., 1994) e alergênica sobre a pele (Reider et al., 2001; Picman, 1986). Devido a isso, nos Estados Unidos a A. montana é considerada pela FDA como "planta perigosa" e seu uso não é permitido em medicamentos por via oral (Newall; Anderson; Phillipson, 2002).

A medicina tradicional brasileira atribui $o$ nome de arnica também a outras espécies da família Asteraceae, entre elas algumas dos gêneros Solidago e Lychnophora. O uso da S. microglossa como arnica, por exemplo, é muito antigo, estando a mesma inscrita na Farmacopéia Brasileira $1^{\text {a }}$ Edição sob o nome de arnica silvestre (Farmacopéia, 1926; Khawali et al., 1986; Chicourel et al., 1997/1998). Já as espécies de Lychnophora vêm sendo mais recentemente muito usadas e comercializadas. A exploração comercial da espécie $L$. ericoides, por exemplo, faz com que a mesma seja até considerada vulnerável (em perigo de extinção) pela Sociedade Brasileira de Botânica (Cabral; Lopes; Lopes, 2000). Estudos farmacológicos confirmaram a atividade antiinflamatória de extratos de $L$. ericoides (Cerqueira, 1997; Borsato et al., 2000) e esta ação foi atribuída à presença de flavonóides, triterpenos e lactonas sesquiterpênicas (Borella et al., 1998). Mais recentemente, Santos et al. (2005) relacionaram a atividade analgésica de extratos hidroalcoólicos da planta à presença de ácidos caféicos. O conjunto desses resultados parece justificar a vasta utilização popular dessa espécie como sucedâneo da A. montana.

Em Minas Gerais, Lychnophora pinaster Mart e L. rupestris Semir \& Leitão-Filho são as mais utilizadas como sucedâneo da $A$. montana. Nessa pesquisa foram determinadas as características físico-químicas e químicas de tinturas fitoterápicas $(\mathrm{TF})$ e homeopáticas (TH) preparadas com essas espécies, em comparação com outras preparadas com A. montana. As tinturas foram então submetidas a estudo de estabilidade, a partir da sua permanência em estufa climatizada por seis meses e em prateleira por 10 meses. O objetivo da pesquisa foi verificar as semelhanças entre essas preparações e verificar as alterações sofridas com o tempo, de forma a contribuir no seu controle de qualidade.

\section{MATERIAIS E MÉTODOS}

\section{Material botânico}

Arnica montana L.: Capítulos florais secos e rasurados de $A$. montana foram adquiridas da Farmácia Reformhaus wieland Kolle, Osterode am Harz (Alemanha), em junho de 2001.

Lychnophora pinaster Mart.: Partes aéreas de L. pinaster Mart. foram coletadas no município de Catas Altas (MG), em 2001 e identificada pelo Prof. João R. Stehmann, do Departamento de Botânica do ICB/UFMG (BHCB 28335).

Lychnophora rupestris Semir \& Leitão-Filho:
Partes aéreas de L. rupestris Semir \& Leitão-Filho foram coletadas no município de Cardeal Mota (MG), em setembro de 2001 e identificadas pelo Prof. João Semir (Unicamp) (BHCB 47135).

\section{Preparação das tinturas}

Após secagem, os materiais foram moídos em moinhos de facas e a granulometria foi determinada. As tinturas fitoterápicas (TF) foram preparadas por percolação, em solução hidroalcoólica a 70\% (V/V), na concentração de $20 \%(\mathrm{p} / \mathrm{V})$ da droga. As tinturas homeopáticas (TH) foram preparadas por maceração em solução hidroalcoólica a 70\% (V/V) por 20 dias, na concentração de $10 \%$ (p/V) da droga (Farm. Hom. Bras. II, 1997).

\section{Caracterização físico-química e química das tinturas}

Caracterização organoléptica: Foram observados aspectos relacionados à cor, odor e sabor das amostras.

Teor alcoólico: As tinturas foram colocadas em provetas e o teor alcoólico foi determinado por meio de alcoômetro.

Densidade: Foi determinada pelo método do picnômetro.

pH: Foi determinado diretamente em potenciômetro.

Porcentagem de resíduo seco total: Exatamente $1 \mathrm{~mL}$ de cada tintura foi pipetado e transferido para cápsulas de porcelana previamente taradas. As cápsulas foram colocadas em banho-maria até secura e, em seguida, levadas à estufa a $110^{\circ} \mathrm{C}$, até peso constante. Após resfriarem em dessecador, as cápsulas foram pesadas, sendo a porcentagem de resíduo seco calculada.

Perfil em CCD: Foram determinados perfis para lactonas sesquiterpênicas (terpenóides) e flavonóides. Utilizaram-se placas de vidro prontas de sílica-gel (Merck, Ref 105721) e eluentes e reveladores indicados por Wagner \& Bladt (1996): (i) para terpenóides tolueno: acetato de etila (93:7) e revelação com reagente de Zimmerman (ZM); (ii) para flavonóides acetato de etila: ácido acético: ácido fórmico: água (100:11:11:26), revelação com NP/PEG, seguida de observação no UV (365 nm). Como referência, utilizou-se soluções metanólicas de quercetina $(5 \mathrm{mg} / 20 \mathrm{~mL})$ e rutina $(5 \mathrm{mg} / 10$ $\mathrm{mL}$ ), isoladas de Dimorphandra mollis, e de licnofolídeo ( $5 \mathrm{mg} / 20 \mathrm{~mL}$ ), isolada de $L$. rupestris.

Identificação dos marcadores químicos por CLAE: Teve como objetivo verificar a presença de quercetina e licnofolídeo nas tinturas e avaliar a variação no teor das mesmas no decorrer do tempo. Utilizou-se um cromatógrafo a líquido de alta eficiência HP 1100, com detector DAD, em $254 \mathrm{~nm}$, e coluna Chromolith RP-18 (Merck). A fase móvel utilizada para a análise de lactonas sesquiterpênicas foi uma mistura de metanol e água 
(50:50), em modo isocrático, com fluxo de $1 \mathrm{~mL} / \mathrm{min}$. Para a separação dos flavonóides, foi utilizada eluição em gradiente (tabela abaixo), utilizando, como solvente A, acetonitrila contendo $1 \%$ de ácido fosfórico $3,3 \times 10^{-2} \mathrm{M}$ e, como solvente $\mathrm{B}$, ácido fosfórico $3,3 \times 10^{-4} \mathrm{M}$ (fluxo de $1 \mathrm{~mL} / \mathrm{min})$.
No tempo zero, todas as tinturas apresentaram características organolépticas muito semelhantes, apresentando coloração avermelhada, odor amadeirado e sabor ligeiramente amargo. Os teores alcoólicos no tempo zero foram variados sendo os mais baixos observados para as tinturas preparadas com A. montana $(\mathrm{AMTF}=$ $60 \%$ e AMTH $=62 \%$ ) em relação às preparadas com as

\begin{tabular}{c|c|c}
\hline Tempo $(\min )$ & Solvente A $(\%)$ & Solvente B $(\%)$ \\
\hline 1 & 95 & 5 \\
20 & 80 & 20 \\
30 & 70 & 30 \\
40 & 20 & 80 \\
50 & 5 & 95 \\
55 & 95 & 5 \\
\hline
\end{tabular}

\section{Estudo da estabilidade das tinturas}

Em estufa climatizada por seis meses: Trezentos $\mathrm{mL}$ de cada tintura, armazenados em frasco âmbar e hermeticamente fechados, foram colocados em estufa climatizada, à temperatura de $30{ }^{\circ} \mathrm{C}$ e $70 \%$ de umidade relativa (UR). As amostras permaneceram na estufa pelo período máximo de 6 meses. Alíquotas de $20 \mathrm{~mL}$ de cada amostra foram retiradas mensalmente, para a realização dos ensaios físico-químicos e químicos descritos anteriormente.

Em vida de prateleira por dez meses: $10,0 \mathrm{~mL}$ de cada uma das tinturas foram armazenados em vidro âmbar e à temperatura ambiente. Após 10 meses as amostras foram submetidas às mesmas análises físicoquímicas e químicas.

\section{RESULTADOS E DISCUSSÃO}

Nesse estudo, foram determinadas as características físico-químicas e químicas de tinturas fitoterápicas (TF) e homeopáticas (TH) preparadas com flores de Arnica montana (AMTF e AMTH), partes aéreas de Lychnophora pinaster (LPTF e LPTH) e $L$. rupestris (LRTF e LRTH). Cada uma das características foi determinada antes (tempo zero) e após a permanência em estufa climatizada a $30^{\circ} \mathrm{C}$ e $70 \%$ UR, por seis meses, e em prateleira por 10 meses. espécies de Lychnophora (LPTF e LRTF $=63 \%$; LRTH $=65 \%$ e LPTH $=67 \%$ ). Os valores de densidade e $\mathrm{pH}$ também variaram no tempo zero entre as tinturas de cada espécie, sendo que as preparadas com A. montana apresentaram valores mais elevados (Tabela 1 ). As TFs apresentaram porcentagem de resíduo seco maiores que as THs, conforme o esperado, mas LPTF e LPTH apresentaram porcentagem de resíduo muito superiores as preparadas com as outras espécies (Tabela 1).

A Farmacopéia Européia descreve que as flores da $A$. montana devem apresentar, no mínimo, $0,4 \%(p / p)$ de lactonas sesquiterpênicas totais, sendo aproximadamente $50 \%$ de helenalina e $11 \alpha, 13$-diidrohelenalina (European, 2001). Lactonas sesquiterpênicas também estão presentes em espécies de Lychnophora. Das partes aéreas de $L$. pinaster foram identificados 15-desoxigoiazensolídeo e ácido licnofóico (Duarte, 1999), enquanto em L. rupestris foram identificadas licnofolido, eremantolido C, eremantina, 15-acetoxi-eremantolido C e 15-acetoxy11(13)-diidroeremantina (Cunha et al., 1995). O perfil cromatográfico obtido para terpenóides em AMTF e AMTH apresentou quatro manchas cinzas com o reagente de ZM, nos Rfs 0,2, 0,32, 0,43 e 0,61, atribuídas às lactonas sesquiterpênicas do tipo helenalina e $11 \alpha, 13$ diidrohelenalina (Wagner; Bladt, 1996). As amostras LRTF e LRTH apresentaram duas manchas cinzas nos Rfs 0,55 e 0,70 , atribuídas à essa mesma classe de substâncias. Essas manchas não foram detectadas em LPTF e LPTH.

Tabela 1. Características físico-químicas das tinturas fitoterápicas (TF) e homeopáticas (TH) preparadas com as três espécis de Arnicas no tempo zero, após seis meses em estufa climatizada e dez meses em prateleira.

\begin{tabular}{c|c|c|c|c|c|c|c|c|c|}
\hline \multirow{2}{*}{ TINTURAS } & \multicolumn{3}{|c|}{ TEMPO ZERO } & \multicolumn{3}{c|}{ SEIS MESES EM ESTUFA } & \multicolumn{2}{c|}{ DEZ MESES EM PRATELEIRA } \\
\cline { 2 - 8 } & DENSIDADE & PH & RESÍDUO SECO & DENSIDADE & PH & RESÍDUO SECO & DENSIDADE & PH & RESIDUO SECO \\
\hline AMTF & 0,9203 & 5,66 & $4,78( \pm 0,32)$ & 0,9254 & 5,03 & $2,06( \pm 0,18)$ & 0,9173 & 5,22 & $2,71( \pm 0,12)$ \\
LPTF & 0,9119 & 5,47 & $5,99( \pm 1,21)$ & 0,9176 & 5,19 & $1,95( \pm 0,44)$ & 0,9142 & 5,31 \\
LRTF & 0,9087 & 5,49 & $3,34( \pm 0,46)$ & 0,9120 & 5,09 & $1,31( \pm 0,53)$ & 0,9132 & 5,28 \\
AMTH & 0,9197 & 5,61 & $1,97( \pm 0,90)$ & 0,9054 & 5,49 & $1,06( \pm 0,30)$ & 0,9092 & 5,50 \\
LPTH & 0,8934 & 5,44 & $2,45( \pm 0,28)$ & 0,8999 & 5,22 & $1,25( \pm 0,25)$ & 0,8942 & 5,40 & $1,33( \pm 0,23)$ \\
LRTH & 0,8994 & 5,46 & $1,60( \pm 0,40)$ & 0,9118 & 5,28 & $1,09( \pm 0,09)$ & 0,9012 & 5,22 \\
\hline
\end{tabular}

$\mathrm{AMTF}=$ A. montana tintura fitoterápica, $\mathrm{LPTF}=L$. pinaster tintura fitoterápica, $\mathrm{LRTF}=L$. rupestris tintura fitoterápica, $\mathrm{AMTH}=$ A. montana tintura homeopática, $\mathrm{LPTH}=L$. pinater tintura homeopática e $\mathrm{LRTH}=L$. rupestris tintura homeopática. 
Para os flavonóides, a Farmacopéia Européia especifica que o teor em A. montana deve se encontrar entre $0,4 \%$ e $0,6 \%$, sendo a quercetina, campferol, patuletina e 6-metoxicampferol os mais importantes. A planta deve conter também até $1 \%$ de ácidos carboxílicos (clorogênico e caféico) e cumarinas, sendo a umbeliferona e escopoletina as mais importantes (Blumenthal, 1998).

Diversos flavonóides e ácidos orgânicos também já foram identificados em espécies de Lychnophora, entre eles ácido cafeico, ácido isoclorogênico, vitexina, isovitexina e quercetina (Silveira et al., 2005). Na análise em CCD no tempo zero, todas as tinturas apresentaram manchas características para flavonóides nos Rfs 0,50 , e 0,30 , além de duas manchas azuis nos $\mathrm{Rfs} 0,85$ e 0,75 , atribuídas aos ácidos caféico e isoclorogênico, respectivamente (Wagner; Bladt, 1996). LPTF e LPTH apresentaram perfis cromatográficos para flavonóides muito semelhantes aos apresentados por AMTF e AMTH, tendo sido observada a presença de manchas atribuídas à rutina e quercetina. Essas substâncias não foram observadas em L. rupestris. As tinturas de L. pinaster diferenciaram-se das demais por apresentarem manchas de coloração azul nos Rf 0,60 e amarela no Rf 0,40. Os resultados demonstram diferenças importantes nos perfis em CCD para lactonas sesquiterpênicas, flavonóides e ácidos orgânicos entre as tinturas de arnica, o que contribui diretamente na identificação das mesmas no controle de qualidade.

A estabilidade é a capacidade de uma formulação manter-se dentro das especificações físico-químicas, microbiológicas, terapêuticas e toxicológicas ao longo do tempo. Vários fatores, como temperatura, umidade, radiação, luz, ar (especificamente oxigênio, dióxido de carbono e vapor de água), $\mathrm{pH}$, propriedades dos solventes, recipientes (frascos de acondicionamento) e a presença de outras substâncias químicas contaminantes podem afetar qualquer componente de uma fórmula farmacêutica, influindo na sua estabilidade (Lachman; Luka; Akers, 1986). Dentre esses, temperatura e umidade são os principais fatores de instabilidade das formulações, pois podem facilmente induzir a degradação das substâncias, mesmo em curto prazo (Brasil, 2001).

No caso das tinturas em análise, as colorações, odores e sabores característicos apresentaram-se menos intensas após o teste de estabilidade. Este comportamento foi mais acentuado para as TFs em relação às TMs. As

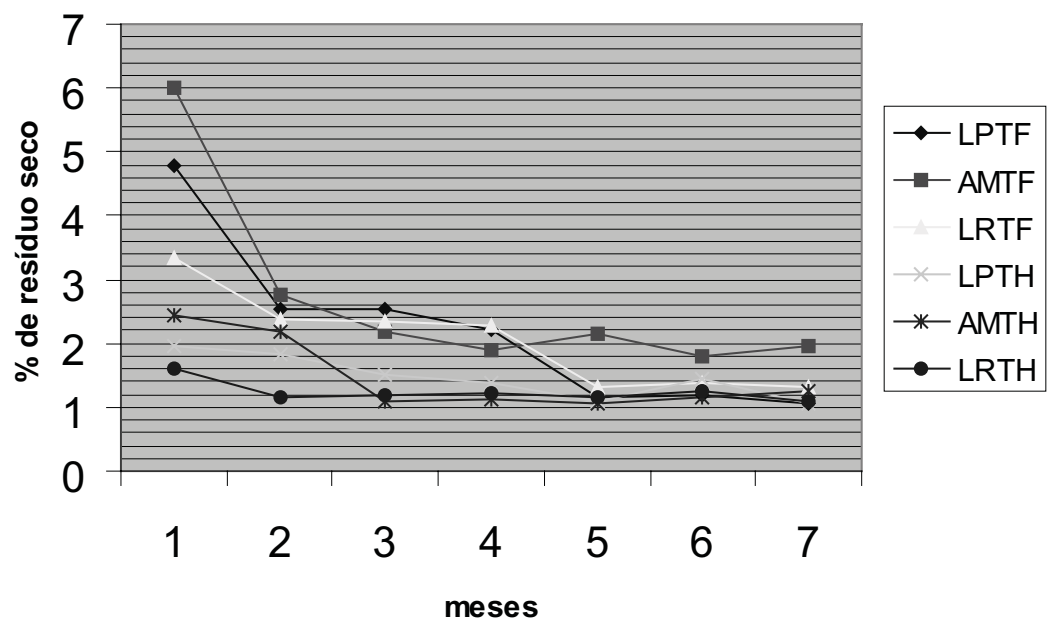

Figura 1. Redução da \% de resíduo seco ao longo dos seis meses em estufa
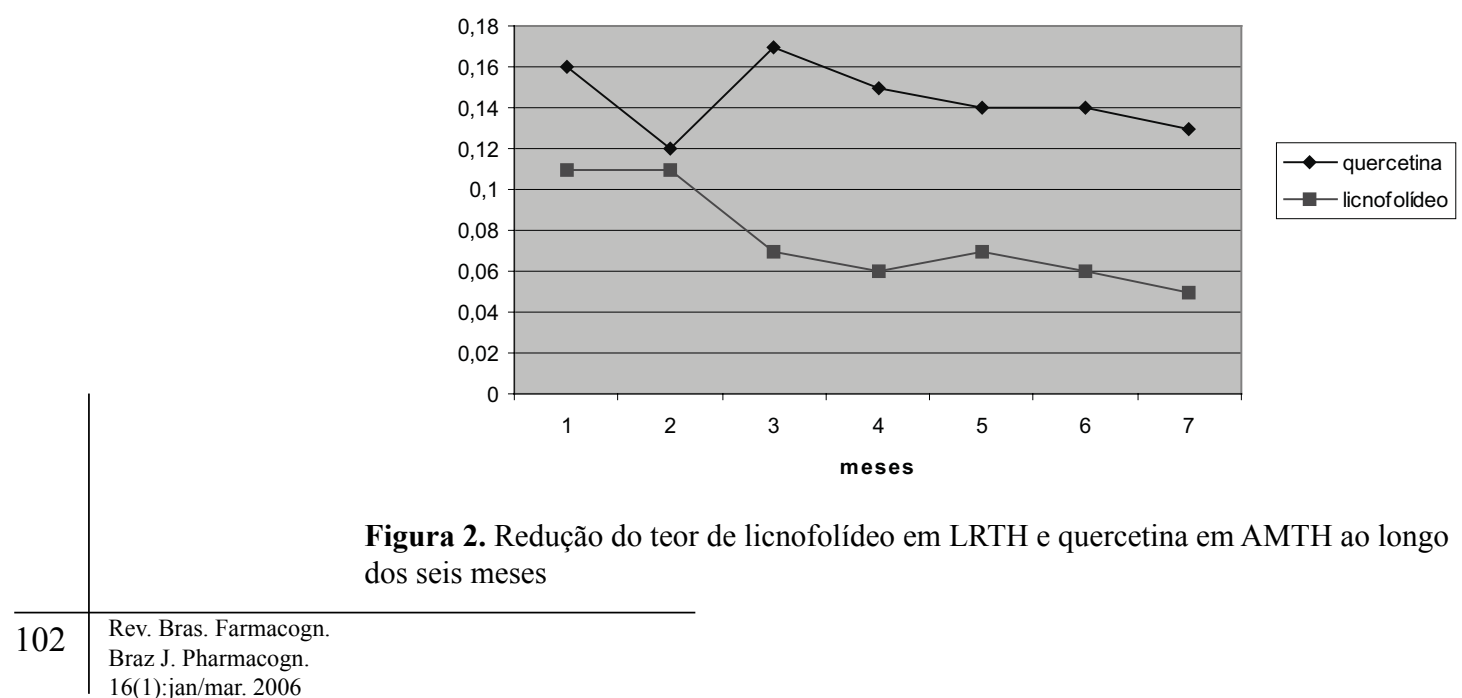

Figura 2. Redução do teor de licnofolídeo em LRTH e quercetina em AMTH ao longo dos seis meses 
demais alterações sofridas encontram-se descritas na Tabela 1. Observou-se, para a maioria das tinturas, uma tendência ao aumento da densidade, conseqüência provável de perda de certa quantidade de solvente, por evaporação. Já os valores de $\mathrm{pH}$ foram reduzidos devido, provavelmente, à degradação química das substâncias.

As mudanças mais significativas, ao final de cada período, foram as reduções das porcentagens de resíduo seco, principalmente após a permanência em estufa a $30^{\circ} \mathrm{C}$ e 70\% (UR) por seis meses (Tabela 1). As porcentagens de perda de massa para as AMTF, LPTF e LRTF foram de $56,90 \%, 67,45 \%$ e $69,78 \%$, respectivamente. Para as THs, as porcentagens foram de 46,18\% para AMTH, $48,98 \%$ para LPTH e $32,0 \%$ para LRTH. A Figura 1 ilustra a perda progressiva de massa ao longo dos seis meses, para em cada uma das tinturas. Observa-se uma expressiva perda de massa já a partir do primeiro mês de armazenamento, revelando a rapidez com que o produto se degrada, naquelas condições.

Os perfis em CCD, por outro lado, não apresentaram diferenças significativas ao final dos testes. Foram observadas as mesmas manchas com as mesmas intensidades que no tempo zero. A degradação dos marcadores químicos foi confirmada nas análises em CLAE. Nas condições cromatográficas utilizadas, a quercetina apresentou tempo de retenção de cerca de 26 minutos e o licnofolídeo de 9 minutos. A partir da medida da área dos picos para quercetina no perfil cromatográfico de AMTH e licnofolídeo em LRTH foi possível observar o declínio na concentração dessas substâncias ao longo do tempo. Após o período de seis meses, a porcentagem de quercetina era de $81 \%$ em relação à concentração original, enquanto a de licnofolídeo chegou a $45,5 \%$ (figura 2). A bibliografia descreve que, no caso dos produtos fitoterápicos, os constituintes terapêuticamente ativos devem apresentar um limite de $\pm 10 \%$ em relação à concentração inicial, após o estudo da estabilidade (Bilia et al., 2001; Schmidt; Matthiensen; Willuhn, 2000). Apesar da cinética da degradação não haver sido determinada nesta pesquisa (é objeto de estudos posteriores), os resultados obtidos já indicam o quanto as tinturas de arnica são instáveis no decorrer do tempo.

\section{CONCLUSÃO}

Os resultados da pesquisa demonstraram diferenças importantes entre as tinturas de arnica que contribuem diretamente na sua identificação e controle de qualidade. Foi demonstrado também que, nas condições de calor úmido, todas as preparações apresentam algum problema de estabilidade, já no primeiro mês de armazenamento. As tinturas armazenadas em condições de vida de prateleira apresentaram alterações menos marcantes. A análise em CCD não permitiu verificar a degradação dos marcadores químicos, que pôde ser detectada apenas por CLAE. O conjunto dos resultados demonstra a instabilidade de extratos vegetais ao longo do tempo e confirma a necessidade de se determinar prazos de validade para os mesmos.

\section{AGRADECIMENTOS}

Ao Prof. João Calegari (USP, Ribeirão Preto) pela doação da amostra de licnofolídeo utilizada como padrão de referência, ao Prof. João Stehmann (ICB/ UFMG) pela indentificação da amostra de L. pinaster e ao Prof. João Semir (UNICAMP) pela identificação da amostra de $L$. rupestris.

\section{REFERÊNCIAS}

Bilia AR, Bergonzi MC, Morgenni F, Mazzi G, Vincieri FF 2001. Evaluation of chemical stability of St. John's wort commercial extract and some preparations. Int J Pharm 213: 199-208.

Blumenthal M 1998. Arnica flowers. In: The complete german commission e monographs: Therapeutic guide to herbal medicines. Austin: American Botanical Council.

Borella JC, Lopes JLC, Vichnewski W, Cunha WR, Herz W 1988. Sesquiterpene lactones, triterpenes and flavones from Lychnophora ericoides and Lychnophora pseudovillosissima. Biochem Syst Ecol 26: 671-676.

Borsato MLC, Grael CFF, Souza GEP, Lopes NP 2000. Analgesic activity of the lignans from Lychnophora ericoides. Phytochemistry 55: 809-813.

Brasil 2001. Agência Nacional de Vigilância Sanitária. Resolução RDC n ${ }^{\circ} 10$, de 2 de janeiro de 2001. Diário Oficial da República Federativa do Brasil, Poder Executivo, Brasília.

Cabral ACS, Lopes NP, Lopes JLC 2000. Terpenóides do tronco de Lychnophora ericoides Mart. XVI Simpósio de Plantas Medicinais do Brasil. Recife, Brasil.

Cerqueira MBS 1987. Ação analgésica do extrato bruto aquoso liofilizado do caule e folhas da Lychnophora ericoides Mart. (arnica). Cienc Cult 39 (5/6): 551553.

Chicourel EL, Pimenta DS, Jorge LIF, Ferro VO 1997/1998. Contribuição ao conhecimento analítico de três Compostas medicinais. Rev Bras Farmacogn 7/8: 59-66.

Cunha WR, Lopes JLC, Vichnewski W, Diaz JG, HERZ W 1995. Eremantholides and a guaianolide from Lychnophora rupestris. Phytochemistry 39: 387389.

Duarte DS 1999. Estudo químico biomonitorado de Lychnophora pinaster Mart. (Asteraceae) e de Macfadyena unguiscati L. A. Gentry (Bignoniaceae)., Belo Horizonte. 177p. Tese de Doutorado - Departamento de Química do Instituto de Ciências Exatas, Universidade Federal de Minas Gerais.

European Pharmacopoeia 2001. 4.ed. European Department for Qualily of Medicines.

Farmacopéia dos Estados Unidos do Brasil 1926. 1 ${ }^{\text {a }}$ Ed. São Paulo: Cia Editora Nacional, 1149p.

Farmacopéia Brasileira 1959. 2a Ed. São Paulo: Indústria Gráfica Siqueira, 1265p.

Farmacopéia Brasileira 1977. $3^{\text {a }}$ Ed. São Paulo: Andrei, 1213. 
Farmacopéia Brasileira 1988-1996. 4ª Ed. São Paulo: Atheneu, $1213 p$.

Farmacopéia Homeopática Brasileira 1997. 2a Ed. São Paulo: Andrei.

Hall IH 1979. Anti-inflammatory activity of sesquiterpene lactones and related compounds. J Pharm Sci 68: 537-542.

Khawali MAH, Akisue MK, Akisue G 1986. Contribuição ao estudo de Solidago microglossa D.C. Rev Bras Farmacogn 1(Supl 1): 55.

Klass CA 2002. Studies on the anti-inflammatory activity of phytopharmaceutics prepared from Arnica flowers. Planta Med 68: 385-391.

Lyss G, Knorre A, Schmidt TJ, Pahl HL, Merfort I 1997. Helenalin, an anti-inflammatory sesquiterpene lactone from Arnica, selectively inhibits transcription factor NF-kappa B. Boll Chim Farmac 378: 951-961.

Newall CA, Anderson LA, Phillipson JD 2002. Arnica. In: Plantas medicinais: guia para profissional de saúde. São Paulo: Premier, p. 40-41.

Picman AK 1986. Biological activities of sesquiterpenes lactones. Biochem Syst Ecol 14: 255-281.

Reider N, Komericki P, Hausen BM, Fritsch P, Aberer W 2001. The seamy side of natural medicines: contact sensitization to arnica (Arnica montana L.) and marigold (Calendula officinalis L.). Contact Dermatitis 45: 269-272.

Rüngeler P, Castro V, Mora G, Gören N, Vichnewski W, Pahl HL, Merfort I, Schmidt TJ 1999. Inhibition of transcription factor NF-kB by sesquiterpene latones: a proposed molecular mechanism of action. Bioorg Med Chem 7: 2342-2352.

Santos MD, Gobbo-Neto L, Albarella L, Souza GEP, Lopes NP 2005. Analgesic activity of di-caffeoylquinic acids from roots of Lychnophora ericoides (Arnica da Serra). J Ethnopharmacol 96: 545-549.

Schmidt TJ, Matthiensen U, Willuhn G 2000. On the stability of sesquiterpene lactones in the officinal Arnica tincture of the German Pharmacopoeia. Planta Med 66: 678-681.

Silveira D, Wagner H, Chiari E, Lombardi JA, Assunção AC, Oliveira AB, Raslan DS 2005. Biological activity of the aqueous extract of Lychnophora pinaster Mart. Rev Bras Farmacogn 15: 294-297.

Wagner H, Bladt S 1996. Plant Drug Analysis: a Thin Layer Chromatography Atlas. 2a Ed, Berlin: Springer $384 \mathrm{p}$.

Woerdenbag HJ, Merfort I, Passreiter CM, Schmidt TJ, Willuhn G, van Uden W, Pras N, Konings AW 1994. Cytotoxicity of flavonoids and sesquiterpene lactones from Arnica species against the GLC4 and the COLO 320 cell lines. Planta Med 60: 434-437. 